\title{
Influence of Surface Energy Effects on Elastic Fields of a Layered Elastic Medium under Surface Loading
}

\author{
Supakorn Tirapat, Teerapong Senjuntichai, and Jaroon Rungamornrat \\ Applied Mechanics and Structures Research Unit, Department of Civil Engineering, Faculty of Engineering, \\ Chulalongkorn University, Bangkok 10330, Thailand \\ Correspondence should be addressed to Teerapong Senjuntichai; teerapong.s@chula.ac.th
}

Received 6 November 2016; Accepted 5 February 2017; Published 26 February 2017

Academic Editor: Philip Eisenlohr

Copyright (c) 2017 Supakorn Tirapat et al. This is an open access article distributed under the Creative Commons Attribution License, which permits unrestricted use, distribution, and reproduction in any medium, provided the original work is properly cited.

\begin{abstract}
This paper presents the analysis of a layered elastic half space under the action of axisymmetric surface loading and the influence of the surface energy effects. The boundary value problems for the bulk and the surface are formulated based on classical linear elasticity and a complete Gurtin-Murdoch constitutive relation. An analytical technique using Love's representation and the Hankel integral transform is employed to derive an integral-form solution for both displacement and stress fields. An efficient numerical quadrature is then applied to accurately evaluate all involved integrals. Selected numerical results are presented to portray the influence of various parameters on elastic fields. Numerical results indicate that the surface stress displays a significant influence on both displacement and stress fields. It is also found that the layered half space becomes stiffer with the presence of surface stresses. In addition, unlike the classical elasticity solution, size-dependent behavior of elastic fields is noted. The present analytical solutions provide fundamental understanding of the influence of surface energy on layered elastic materials. It can also be used as a benchmark solution for the development of numerical techniques such as FEM and BEM, for analysis of more complex problems involving a layered medium under the influence of surface energy effects.
\end{abstract}

\section{Introduction}

Nanotechnology has received wide attention in recent years due to its vast applications in various disciplines such as biology, chemistry, physics, medicines, material sciences, and engineering. In the fields of material sciences and engineering, studies related to mechanical behavior of nanostructured materials have become a subject of numerous investigations due to the fact that understanding fundamental aspects of their behaviors at nanoscale level is important for optimum design of nanosized devices and structures. There are two approaches that have commonly been employed to theoretically investigate mechanical behaviors of materials at nanoscale, namely, atomistic simulation and modified continuum-based model. Atomistic modeling techniques require a very large computational effort, although they are considered very accurate. A modified continuum-based model then becomes an attractive alternative in obtaining first-approximation to predict mechanical behaviors of nanostructured materials. Due to their high surfaceto-volume ratio, nanoscale elements usually exhibit high influence of surface/interface free energy, which is the energy associated with atoms at or near a free surface (e.g., see [1]); consequently, their mechanical behavior becomes size-dependent [2]. Thus, surface energy effects, which are generally ignored in conventional continuum mechanics problems, need to be taken into account in modified continuum-based simulation for nanoscale systems. A theoretical framework based on continuum mechanics concepts was proposed by Gurtin and Murdoch [3, 4] to take into consideration the influence of surface energy effects. In their model, an elastic surface was formed as a mathematical layer of zero thickness perfectly bonded to the underlying bulk material without slipping. Several studies were carried out to verify that modified continuum-based simulations with surface energy effects and size-dependency can be employed to model nanostructured elements with acceptable accuracy. For instance, Miller and Shenoy [5] examined the sizedependent behavior of nanostructured elements (i.e., bar, beam, and plate) by adopting the Gurtin-Murdoch model and found that their results were in a good agreement with those 
obtained from direct atomistic simulations. Dingreville et al. [6] developed a continuum framework to incorporate the surface free energy in the framework of continuum mechanics and demonstrated that overall mechanical behaviors of nanostructured elements such as particles, wires, and films were found to be size-dependent. There also exist other continuum-based theories that have been developed to take into account the size-dependent material behaviors at the nanoscale level such as the strain gradient elasticity theory by Mindlin [7]. The theory proposed by Mindlin has not been widely adopted in the modeling of nanoscale systems since it involves several additional material parameters and higherorder governing equations. Simplified versions of Mindlin's theory have then been proposed, and analytical solutions to various continuum mechanics problems were presented based on its simplified versions (e.g., see [8-10]).

Over the last two decades, several researchers have investigated a variety of continuum mechanics problems by adopting the Gurtin-Murdoch theory of surface elasticity. For example, Huang and $\mathrm{Yu}$ [11] studied an elastic half plane under surface loading with consideration of surface energy effects. An elastic layer with finite thickness, subjected to surface loading under plane-strain and axisymmetric conditions, was also considered by Zhao and Rajapakse [12]. Intarit et al. [13] derived fundamental solutions of an elastic half plane under internal loading and dislocations. An elastic half plane under surface shear loading was also investigated by Lei et al. [14]. Recently, a nanocontact problem of layered viscoelastic solids with surface energy effects was presented by Abdel Rahman and Mahmoud [15]. All these studies, however, considered the surface stress tensor as a $2 \mathrm{D}$ quantity with its out-of-plane components being neglected. Wang et al. [16] showed that the out-of-plane terms of the surface displacement gradient could be significant even in the case of small deformations particularly for curved and rotated surfaces. The complete version of Gurtin-Murdoch model, with consideration of the out-of-plane term, has later been employed to examine various continuum mechanics problems, for example, problems related to an internally loaded elastic layer under plane-strain condition [17] and axisymmetric loading [18], respectively; contact problem [19]; nanoindentation [20, 21]; nanobeams [22]; nanoplate [23]; and nanosized cracks $[24,25]$. In addition, the influence of surface energy is also significant in problems related to soft elastic solids [26].

Stress analysis of a layered elastic medium under applied surface loading has a rich history (e.g., see [27-30]) due to its close relevance to various engineering applications, such as characterization of mechanical properties of layered materials: for example, protective coatings, multilayer capacitors, and layered composite materials; analysis and design of pavement and foundations; and in situ testing of soils and rocks and so forth. A review of literature indicates that studies related to a layered elastic medium with consideration of surface energy effects based on the Gurtin-Murdoch theory are very limited. This class of problems has extensive applications in the study of nanocoatings and nanoscale surface layers that are used in electronic devices, tribological and biomaterial applications, advanced industrial materials,

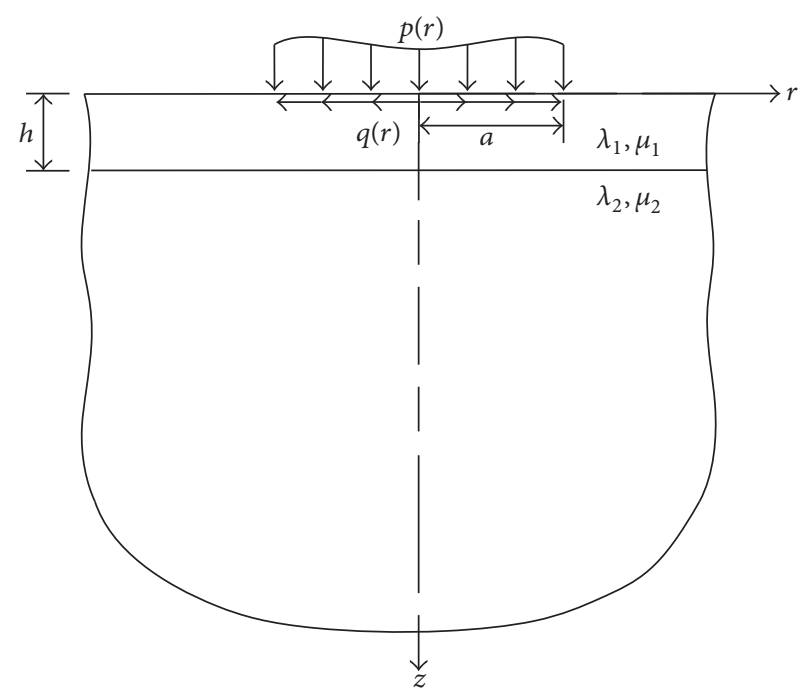

FIGURE 1: Layered elastic half space subjected to axisymmetric surface loading.

communication devices, and so forth. The main objective of this paper is to present analytical solutions to a layered elastic half space under axisymmetric surface loading by adopting the complete Gurtin-Murdoch theory of surface elasticity. The boundary value problems of a layered elastic half space under axisymmetric surface loading involving nonclassical boundary conditions due to surface stress influence are formulated by employing Love's strain potential and the Hankel integral transform. Selected numerical results for displacements and stresses due to applied vertical and radial loading are presented to portray the influence of layer thickness, surface material parameters, and size-dependency on elastic fields. The present fundamental solution is useful in the development of boundary integral equation methods for the investigation of more complicated problems such as nanoindentation and contact problems involving a layered elastic medium. In addition, the present numerical results can also be employed as a benchmark solution in the development of numerical techniques such as finite element and boundary element methods for analysis of a variety of problems with the influence of surface energy such as nanoscale problems and soft elastic solids.

\section{Governing Equations and General Solutions}

Consider a layered elastic half space consisting of two elastic materials with different properties perfectly bonded together, in which the upper material is an elastic layer of finite thickness $h$ and subjected to axisymmetric vertical and radial surface loads denoted by $p(r)$ and $q(r)$, respectively, as shown in Figure 1. According to the Gurtin-Murdoch surface elasticity theory, both materials consist of two parts, the bulk material and the surface, which is a zero-thickness layer perfectly bonded to the bulk material without slipping. The equilibrium equations, the constitutive equations, and the strain-displacement relationship of the bulk material under 
axisymmetric deformations are the same as those in the classical elasticity theory, which are given, respectively, by

$$
\begin{aligned}
\frac{\partial \sigma_{r r}}{\partial r}+\frac{\partial \sigma_{r z}}{\partial z}+\frac{\sigma_{r r}-\sigma_{\theta \theta}}{r} & =0 \\
\frac{\partial \sigma_{r z}}{\partial r}+\frac{\partial \sigma_{z z}}{\partial z}+\frac{\sigma_{r z}}{r} & =0 \\
\sigma_{r r} & =(\lambda+2 \mu) \varepsilon_{r r}+\lambda \varepsilon_{\theta \theta}+\lambda \varepsilon_{z z}, \\
\sigma_{\theta \theta} & =\lambda \varepsilon_{r r}+(\lambda+2 \mu) \varepsilon_{\theta \theta}+\lambda \varepsilon_{z z}, \\
\sigma_{z z} & =\lambda \varepsilon_{r r}+\lambda \varepsilon_{\theta \theta}+(\lambda+2 \mu) \varepsilon_{z z}, \\
\sigma_{r z} & =2 \mu \varepsilon_{r z}, \\
\varepsilon_{r r} & =\frac{\partial u_{r}}{\partial r}, \\
\varepsilon_{\theta \theta} & =\frac{u_{r}}{r}, \\
\varepsilon_{z z} & =\frac{\partial u_{z}}{\partial z}, \\
\varepsilon_{r z} & =\frac{1}{2}\left(\frac{\partial u_{r}}{\partial z}+\frac{\partial u_{z}}{\partial r}\right),
\end{aligned}
$$

where $\left\{\sigma_{r r}, \sigma_{\theta \theta}, \sigma_{z z}, \sigma_{r z}\right\}$ denote the components of stress tensors; $\left\{\varepsilon_{r r}, \varepsilon_{\theta \theta}, \varepsilon_{z z}, \varepsilon_{r z}\right\}$ denote the components of strain tensors; and $\left\{u_{r}, u_{z}\right\}$ denote the components of displacement tensors, respectively. In addition, $\mu$ and $\lambda$ are Lamé constants of a bulk material. On the surface, the equilibrium conditions in terms of the generalized Young-Laplace equation [31], the surface constitutive relations, and the strain-displacement relationship can be expressed, respectively, as [3,4]

$$
\begin{aligned}
& \frac{\partial \sigma_{r r}^{s}}{\partial r}+\frac{\sigma_{r r}^{s}-\sigma_{\theta \theta}^{s}}{r}+\left.\sigma_{z r}\right|_{z=0}+t_{r}^{0}=0, \\
& \frac{\partial \sigma_{z r}^{s}}{\partial r}+\frac{\sigma_{z r}^{s}}{r}+\left.\sigma_{z z}\right|_{z=0}+t_{z}^{0}=0, \\
& \sigma_{r r}^{s}=\tau^{s}+\left(2 \mu^{s}+\lambda^{s}\right) \varepsilon_{r r}^{s}+\left(\lambda^{s}+\tau^{s}\right) \varepsilon_{\theta \theta}^{s}, \\
& \sigma_{\theta \theta}^{s}=\tau^{s}+\left(2 \mu^{s}+\lambda^{s}\right) \varepsilon_{\theta \theta}^{s}+\left(\lambda^{s}+\tau^{s}\right) \varepsilon_{r r}^{s}, \\
& \sigma_{z r}^{s}=\tau^{s} \frac{d u_{z}^{s}}{d r}, \\
& \varepsilon_{r r}^{s}=\frac{d u_{r}^{s}}{d r}, \\
& \varepsilon_{\theta \theta}^{s}=\frac{u_{r}^{s}}{r},
\end{aligned}
$$

where the superscript " $s$ " is used to denote the quantities corresponding to the surface; $\lambda^{s}$ and $\mu^{s}$ are surface Lamé constants; $\tau^{s}$ is the residual surface stress (or surface tension) under unstrained conditions; and $t^{0}$ denotes the prescribed traction on the surface. Equation (4) can be viewed as the outof-plane contribution of the preexisting surface tension $\tau^{s}$ in the deformed configuration whereas the surface gradient of the displacement $d u_{z}^{s} / d r$ acts as the out-of-plane component of the unit vector tangent to the surface in the deformed state. This term has been ignored in several previous studies even though the contribution of $\tau^{s}$ could be significant even in the case of small deformations (e.g., see [17, 18, 20]).

The general solutions for the stresses and displacements in the bulk material under axisymmetric deformations can be expressed by using Love's strain potential and Hankel integral transform as [32]

$$
\begin{aligned}
\sigma_{r r} & =\int_{0}^{\infty} \xi\left[\lambda \frac{d^{3} \Omega}{d z^{3}}+(\lambda+2 \mu) \xi^{2} \frac{d \Omega}{d z}\right] J_{0}(\xi r) d \xi \\
& -\frac{2(\lambda+\mu)}{r} \int_{0}^{\infty} \xi^{2} \frac{d \Omega}{d z} J_{1}(\xi r) d \xi \\
\sigma_{\theta \theta} & =\lambda \int_{0}^{\infty} \xi\left[\frac{d^{3} \Omega}{d z^{3}}-\xi^{2} \frac{d \Omega}{d z}\right] J_{0}(\xi r) d \xi+\frac{2(\lambda+\mu)}{r} \\
& \cdot \int_{0}^{\infty} \xi^{2} \frac{d \Omega}{d z} J_{1}(\xi r) d \xi \\
\sigma_{z z} & =\int_{0}^{\infty} \xi\left[(\lambda+2 \mu) \frac{d^{3} \Omega}{d z^{3}}-(3 \lambda+4 \mu) \xi^{2} \frac{d \Omega}{d z}\right] \\
& \cdot J_{0}(\xi r) d \xi \\
\sigma_{r z} & =\int_{0}^{\infty} \xi\left[\lambda \frac{d^{2} \Omega}{d z^{2}}+(\lambda+2 \mu) \xi^{2} \Omega\right] J_{1}(\xi r) d \xi \\
u_{r} & =\frac{\lambda+\mu}{\mu} \int_{0}^{\infty} \xi^{2} \frac{d \Omega}{d z} J_{1}(\xi r) d \xi, \\
u_{z}= & \int_{0}^{\infty} \xi\left[\frac{d^{2} \Omega}{d z^{2}}-\frac{\lambda+2 \mu}{\mu} \xi^{2} \Omega\right] J_{0}(\xi r) d \xi,
\end{aligned}
$$

where

$$
\Omega(\xi, z)=(A+B z) e^{-\xi z}+(C+D z) e^{\xi z} .
$$

In addition, $A, B, C$, and $D$ are the arbitrary functions to be determined from the appropriate boundary conditions.

\section{Solutions for Axisymmetric Surface Loading}

Boundary value problem of a layered elastic half space subjected to axisymmetric normal and tangential traction, denoted by $p(r)$ and $q(r)$, respectively, applied at its surface as shown in Figure 1 is considered in this section. To solve this problem, the layered half space is divided into two domains. Domain " 1 " represents the upper layer and domain " 2 " represents the underlying half space. The general solutions of the bulk material in domain " 1 " are given by (6) whereas those of domain " 2 " can also be obtained from (6) by replacing the arbitrary functions $A$ to $D$ with the arbitrary functions $E$ to $H$, respectively. Note that $G \equiv 0$ and $H \equiv 0$ are imposed to ensure the regularity of the solutions at infinity for domain " 2 "; in addition, the subscript $i=1,2$ is used to denote the quantities corresponding to domains " 1 " and " 2 ," respectively. 
The solutions of $A$ to $F$ can be determined by solving the following boundary and continuity conditions:

$$
\begin{aligned}
& \left.\sigma_{z z 1}\right|_{z=0}+\tau_{1}^{s}\left(\frac{d^{2} u_{z 1}}{d r^{2}}+\frac{1}{r} \frac{d u_{z 1}}{d r}\right)_{z=0}=-p(r), \\
& \left.\sigma_{r z 1}\right|_{z=0}+\kappa_{1}^{s}\left(\frac{d^{2} u_{r 1}}{d r^{2}}+\frac{1}{r} \frac{d u_{r 1}}{d r}-\frac{u_{r 1}}{r^{2}}\right)_{z=0}=-q(r), \\
& \left\{\left.\sigma_{z z 1}\right|_{z=h}+\tau_{1}^{s}\left(\frac{d^{2} u_{z 1}}{d r^{2}}+\frac{1}{r} \frac{d u_{z 1}}{d r}\right)_{z=h}\right\} \\
& -\left\{\left.\sigma_{z z 2}\right|_{z=h}+\tau_{2}^{s}\left(\frac{d^{2} u_{z 2}}{d r^{2}}+\frac{1}{r} \frac{d u_{z 2}}{d r}\right)_{z=h}\right\}=0, \\
& \left\{\left.\sigma_{r z 1}\right|_{z=h}+\kappa_{1}^{s}\left(\frac{d^{2} u_{r 1}}{d r^{2}}+\frac{1}{r} \frac{d u_{r 1}}{d r}-\frac{u_{r 1}}{r^{2}}\right)_{z=h}\right\} \\
& -\left\{\left.\sigma_{r z 2}\right|_{z=h}+\kappa_{2}^{s}\left(\frac{d^{2} u_{r 2}}{d r^{2}}+\frac{1}{r} \frac{d u_{r 2}}{d r}-\frac{u_{r 2}}{r^{2}}\right)\right. \\
& =0, \\
& \left.u_{z 1}\right|_{z=h}-\left.u_{z 2}\right|_{z=h}=0, \\
& \left.u_{r 1}\right|_{z=h}-\left.u_{r 2}\right|_{z=h}=0,
\end{aligned}
$$

where $\kappa_{i}^{s}=2 \mu_{i}^{s}+\lambda_{i}^{s}(i=1,2)$ is a surface material constant. It should be noted that (8) are nonclassical boundary conditions obtained from (2) to (5). In view of (6) together with the assumption that the surface residual stress $\tau^{s}$ is constant, the following six linear algebraic equations are established to solve for the arbitrary functions $A$ to $F$ :

$$
\begin{aligned}
& \left(\bar{\lambda}_{1}+1+\delta_{1} \bar{\xi}\right) A \bar{\xi}+\left(1+\bar{\tau}_{1}^{s} \bar{\xi}\right) B+\left(-\left(\bar{\lambda}_{1}+1\right)+\delta_{1} \bar{\xi}\right) \\
& C \cdot \bar{\xi}+\left(1-\bar{\tau}_{1}^{s} \bar{\xi}\right) D=-\frac{\bar{P}(\bar{\xi})}{2 \bar{\xi}^{2}}, \\
& \left(\left(\bar{\lambda}_{1}+1\right)+\gamma_{1} \bar{\xi}\right) A \bar{\xi}+\left(-\bar{\lambda}_{1}-\gamma_{1} \bar{\xi}\right) B \\
& +\left(\left(\bar{\lambda}_{1}+1\right)-\gamma_{1} \bar{\xi}\right) C \bar{\xi}+\left(\bar{\lambda}_{1}-\gamma_{1} \bar{\xi}\right) D=-\frac{\bar{Q}(\bar{\xi})}{2 \bar{\xi}^{2}}, \\
& \left(\bar{\lambda}_{1}+1\right) e^{-\bar{h} \bar{\xi}} A \bar{\xi}+\left(1+\left(\bar{\lambda}_{1}+1\right) \bar{h} \bar{\xi}\right) e^{-\bar{h} \bar{\xi}} B-\left(\bar{\lambda}_{1}+1\right) \\
& \cdot e^{\bar{h} \bar{\xi}} C \bar{\xi}+\left(1-\left(\bar{\lambda}_{1}+1\right) \bar{h} \bar{\xi}\right) e^{\bar{h} \bar{\xi}} D \\
& +\left(-\left(\bar{\lambda}_{2}+\bar{\mu}_{2}\right)+\delta_{2} \bar{\xi}\right) e^{-\overline{h \bar{\xi}}} E \bar{\xi} \\
& +\left(-\bar{\mu}_{2}-\left(\bar{\lambda}_{2}+\bar{\mu}_{2}\right) \bar{h} \bar{\xi}+\left(\frac{2 \bar{\mu}_{2}}{\bar{\lambda}_{2}+\bar{\mu}_{2}}+\bar{h} \bar{\xi}\right) \delta_{2} \bar{\xi}\right) \\
& \cdot e^{-\bar{h} \bar{\xi}} F=0
\end{aligned}
$$

$$
\begin{aligned}
& \left(\bar{\lambda}_{1}+1\right) e^{-\bar{h} \bar{\xi}} A \bar{\xi}+\left(-\bar{\lambda}_{1}+\left(\bar{\lambda}_{1}+1\right) \bar{h} \bar{\xi}\right) e^{-\bar{h} \bar{\xi}} B \\
& +\left(\bar{\lambda}_{1}+1\right) e^{\bar{h} \bar{\xi}} C \bar{\xi}+\left(\bar{\lambda}_{1}+\left(\bar{\lambda}_{1}+1\right) \bar{h} \bar{\xi}\right) e^{\bar{h} \bar{\xi}} D \\
& +\left(-\left(\bar{\lambda}_{2}+\bar{\mu}_{2}\right)+\gamma_{2} \bar{\xi}\right) e^{-\overline{h \xi}} E \bar{\xi} \\
& +\left(\bar{\lambda}_{2}-\left(\bar{\lambda}_{2}+\bar{\mu}_{2}\right) \bar{h} \bar{\xi}-(1-\bar{h} \bar{\xi}) \gamma_{2} \bar{\xi}\right) e^{-\bar{h} \bar{\xi}} F=0 \\
& {\left[-\left(\bar{\lambda}_{1}+1\right) e^{-\bar{h} \bar{\xi}}\right] A \bar{\xi}+\left[\left(-2-\left(\bar{\lambda}_{1}+1\right) \bar{h} \bar{\xi}\right) e^{-\bar{h} \bar{\xi}}\right] B} \\
& +\left[-\left(\bar{\lambda}_{1}+1\right) e^{\bar{h} \bar{\xi}}\right] C \bar{\xi}+\left[\left(2-\left(\bar{\lambda}_{1}+1\right) \bar{h} \bar{\xi}\right) e^{\bar{h} \bar{\xi}}\right] D \\
& +\left[\left(\frac{\bar{\lambda}_{2}+\bar{\mu}_{2}}{\bar{\mu}_{2}}\right) e^{-\bar{h} \bar{\xi}}\right] E \bar{\xi} \\
& +\left[\left(2+\left(\frac{\bar{\lambda}_{2}+\bar{\mu}_{2}}{\bar{\mu}_{2}}\right) \bar{h} \bar{\xi}\right) e^{-\bar{h} \bar{\xi}}\right] F=0, \\
& {\left[-\left(\bar{\lambda}_{1}+1\right) e^{-\bar{h} \bar{\xi}}\right] A \bar{\xi}+\left[\left(\bar{\lambda}_{1}+1\right)(1-\bar{h} \bar{\xi}) e^{-\bar{h} \bar{\xi}}\right] B} \\
& +\left[\left(\bar{\lambda}_{1}+1\right) e^{\bar{h} \bar{\xi}}\right] C \bar{\xi}+\left[\left(\bar{\lambda}_{1}+1\right)(1+\bar{h} \bar{\xi}) e^{\bar{h} \bar{\xi}}\right] D \\
& +\left[\left(\frac{\bar{\lambda}_{2}+\bar{\mu}_{2}}{\bar{\mu}_{2}}\right) e^{-\bar{h} \bar{\xi}}\right] E \bar{\xi} \\
& +\left[\left(\frac{\bar{\lambda}_{2}+\bar{\mu}_{2}}{\bar{\mu}_{2}}\right)(-1+\bar{h} \bar{\xi}) e^{-\overline{h \xi}}\right] F=0 \text {, }
\end{aligned}
$$

where the following nondimensional quantities in the above equations are defined thus: $\bar{h}=h / \Lambda_{1} ; \bar{\lambda}_{i}=\lambda_{i} / \mu_{1} ; \bar{\mu}_{2}=\mu_{2} /$ $\mu_{1} ; \delta_{1}=\left(\bar{\lambda}_{1}+1\right) \bar{\tau}_{1}^{s} / 2 ; \delta_{2}=\left(\bar{\lambda}_{2}+\bar{\mu}_{2}\right) \bar{\tau}_{2}^{s} / 2 \bar{\mu}_{2} ; \gamma_{1}=\left(\bar{\lambda}_{1}+\right.$ $1) \bar{\kappa}_{1}^{s} / 2 ; \gamma_{2}=\left(\bar{\lambda}_{2}+\bar{\mu}_{2}\right) \bar{\kappa}_{2}^{s} / 2 \bar{\mu}_{2} ; \bar{\tau}_{1}^{s}=\tau_{1}^{s} / \mu_{1} \Lambda_{1} ; \bar{\tau}_{2}^{s}=\left(\tau_{1}^{s}-\right.$ $\left.\tau_{2}^{s}\right) / \mu_{1} \Lambda_{1} ; \bar{\kappa}_{1}^{s}=\kappa_{1}^{s} / \mu_{1} \Lambda_{1} ; \bar{\kappa}_{2}^{s}=\left(\kappa_{1}^{s}-\kappa_{2}^{s}\right) / \mu_{1} \Lambda_{1} ;$ and $\Lambda_{1}=$ $\kappa_{1}^{s}\left(\lambda_{1}+2 \mu_{1}\right) / 2 \mu_{1}\left(\lambda_{1}+\mu_{1}\right)$. In addition, the functions $\bar{P}(\bar{\xi})$ and $\bar{Q}(\bar{\xi})$ are obtained from the surface loads $p(r)$ and $q(r)$, respectively, as

$$
\begin{aligned}
& \bar{P}(\bar{\xi})=\int_{0}^{\infty} \bar{p}(\bar{r}) J_{0}(\bar{\xi} \bar{r}) \bar{r} d \bar{r} \\
& \bar{Q}(\bar{\xi})=\int_{0}^{\infty} \bar{q}(\bar{r}) J_{1}(\bar{\xi} \bar{r}) \bar{r} d \bar{r}
\end{aligned}
$$

in which $\bar{p}=p / \mu_{1} ; \bar{q}=q / \mu_{1}$; and $\bar{r}=r / \Lambda_{1}$. The arbitrary functions $A$ to $F$ for given functions of the applied surface loads $p(r)$ and $q(r)$ can then be obtained by solving the linear equation system (9). Substitution of the arbitrary functions $A$ to $F$ into (6) yields the displacement and stress fields at an arbitrary point of the layered elastic half space shown in Figure 1.

\section{Numerical Results and Discussion}

The complete solutions of displacements and stresses corresponding to the boundary value problems of a layered 
TABLE 1: Comparison of normalized surface displacements and stresses at the interface of a layered elastic half space under uniformly distributed normal traction for $\mu_{1} / \mu_{2}=5$ and $h / a=1$.

\begin{tabular}{lcccccccc}
\hline \multirow{2}{r}{$/ a$} & \multicolumn{2}{c}{$\mu_{1} u_{z} / a p_{0}$} & \multicolumn{2}{c}{$\mu_{1} u_{r} / a p_{0}$} & \multicolumn{2}{c}{$\sigma_{z z} / p_{0}$} & \multicolumn{2}{c}{$\sigma_{r z} / p_{0}$} \\
& Gerrard [27] & Present solution & Gerrard [27] & Present solution & Gerrard [27] & Present solution & Gerrard [27] & Present solution \\
\hline 0.0 & 0.9945 & 0.9944 & 0.0000 & 0.0000 & 0.4260 & 0.4260 & 0.0000 \\
0.5 & 0.9442 & 0.9440 & -0.0746 & -0.0746 & 0.3790 & 0.3790 & 0.0867 & 0.0000 \\
1.0 & 0.7651 & 0.7649 & -0.1363 & -0.1363 & 0.2526 & 0.2526 & 0.1303 & 0.1303 \\
2.0 & 0.4630 & 0.4629 & -0.1048 & -0.1048 & 0.0657 & 0.0657 & 0.0719 & 0.0719 \\
3.0 & 0.3179 & 0.3177 & -0.0747 & -0.0748 & 0.0174 & 0.0174 & 0.0307 & 0.0307 \\
5.0 & 0.1867 & 0.1866 & -0.0420 & -0.0421 & 0.0010 & 0.0010 & 0.0069 & 0.0069 \\
10.0 & 0.0933 & 0.0932 & -0.0217 & -0.0218 & 0.0001 & 0.0000 & 0.0009 & 0.0009 \\
\hline
\end{tabular}

TABLE 2: Comparison of normalized surface displacements and stresses at the interfaces of a layered elastic half space under linearly distributed shear traction for $\mu_{1} / \mu_{2}=5$ and $h / a=1$.

\begin{tabular}{ccccccccc}
\hline \multirow{2}{r}{$r / a$} & \multicolumn{2}{c}{$\mu_{1} u_{z} / a q_{0}$} & \multicolumn{2}{c}{$\mu_{1} u_{r} / a q_{0}$} & \multicolumn{2}{c}{$\sigma_{z z} / q_{0}$} & \multicolumn{2}{c}{$\sigma_{r z} / q_{0}$} \\
& Gerrard [27] & Present solution & Gerrard [27] & Present solution & Gerrard [27] & Present solution & Gerrard [27] & Present solution \\
\hline 0.0 & 0.1188 & 0.1189 & 0.0000 & 0.0000 & 0.1150 & 0.1150 & 0.0000 & 0.0000 \\
0.5 & 0.0941 & 0.0941 & -0.0952 & -0.0952 & 0.0803 & 0.0803 & 0.0359 & 0.0359 \\
1.0 & 0.0253 & 0.0253 & -0.0934 & -0.0934 & 0.0173 & 0.0173 & 0.0312 & 0.0312 \\
2.0 & 0.0044 & 0.0044 & -0.0198 & -0.0198 & -0.0068 & -0.0068 & -0.0005 & -0.0005 \\
3.0 & -0.0003 & -0.0003 & -0.0087 & -0.0087 & -0.0028 & -0.0028 & -0.0020 & -0.0020 \\
4.0 & -0.0006 & -0.0005 & -0.0051 & -0.0051 & -0.0010 & -0.0010 & -0.0012 & -0.0012 \\
5.0 & -0.0003 & -0.0003 & -0.0036 & -0.0036 & -0.0004 & -0.0004 & -0.0007 & -0.0007 \\
10.0 & 0.0001 & 0.0001 & -0.0014 & -0.0014 & 0.0000 & 0.0000 & -0.0001 & -0.0001 \\
\hline
\end{tabular}

elastic medium under axisymmetric surface loading shown in Figure 1 are given by (6) with the arbitrary functions $A$ to $F$ obtained by solving (9). The solutions of $A$ to $F$ are obtained explicitly for applied normal and tangential traction, $p(r)$ and $q(r)$, respectively, but their expressions for each loading case are not presented here for brevity. Given the complexity of the arbitrary functions $A$ to $F$, closed-form solutions to the displacement and stress fields cannot be obtained. Therefore it is essential to determine all elastic fields by numerically evaluating the semi-infinite integrals appearing in (6). It is found that those semi-infinite integrals with respect to $\xi$ can be accurately evaluated by employing the numerical quadrature scheme based on 21-point GaussKronrod rule [33]. The accuracy of the present solution is first verified by comparing with the existing solution given by Gerrard [27], who presented the classical solutions (without the influence of surface energy effects) of a layered elastic half space subjected to axisymmetric surface loading. Table 1 presents a comparison of normalized displacements at the surface $(z=0)$ and normalized stresses at the interface $(z=h)$ along the radial direction of a layered elastic half space under uniformly distributed normal traction $p_{0}$, acting over a circular area of radius $a$ at the surface. The comparison of surface displacements and stresses at the interface of the layered half space under linearly distributed shear traction $\bar{q}(\bar{r})=-q_{0} r / \mu_{1} a$ applied over a circular area of radius $a$ at the surface is also shown in Table 2 . In addition, $\mu_{1} / \mu_{2}=5$ with Poisson's ratio $\nu_{1}=\nu_{2}=0.2$ and $h / a=1$ are considered for the numerical results presented in both tables. The solutions for normalized displacements and stresses from the present study are obtained by setting the parameters associated with the surface energy effects to be zero; that is, $\tau^{s} \equiv 0$ and $\kappa^{s} \equiv 0$. It is evident that excellent agreement between the two solutions is observed in both displacements and stresses shown in Tables 1 and 2.

Numerical results for vertical and radial displacements and vertical and shear stresses corresponding to a layered elastic half space with the influence of surface energy effects subjected to axisymmetric surface loading as shown in Figure 1 are presented next. Two cases of axisymmetric surface loading, namely, the vertical loading and the radial loading, are considered in the numerical study. The vertical loading denotes the case where uniformly distributed normal traction $p_{0}$ is applied over a circular area of normalized radius $a / \Lambda_{1}=\bar{a}=10$. The radial loading represents the case where the layered half space is subjected to linearly distributed tangential traction $\bar{q}(\bar{r})=q_{0} \bar{r} / \mu_{1} \bar{a}$ over a circular area of normalized radius $\bar{a}=10$, where $q_{0}$ is the maximum traction at the edge of the loading region. The functions defined as shown in (10) are given, respectively, for the vertical loading and the radial loading as follows:

$$
\begin{aligned}
\bar{P}(\bar{\xi}) & =\frac{p_{0} \bar{a}}{\bar{\xi}} J_{1}(\bar{\xi} \bar{a}), \\
\bar{Q} & =0
\end{aligned}
$$



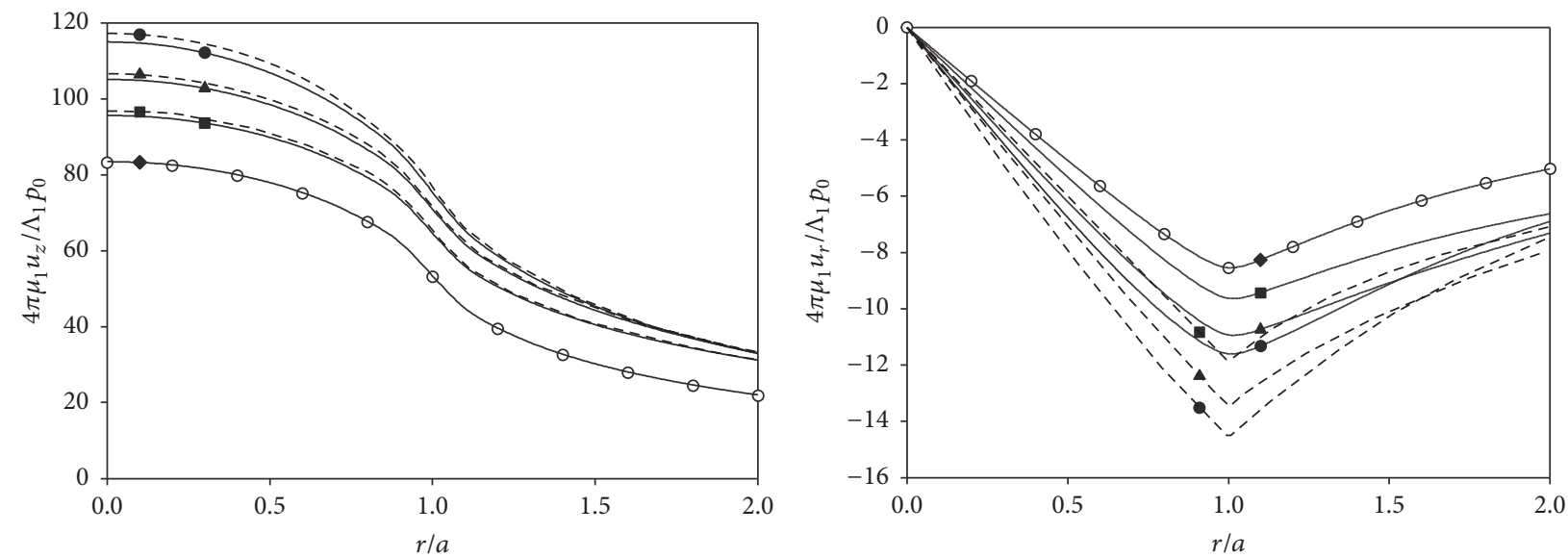

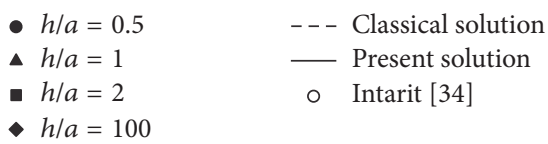

- $h / a=100$

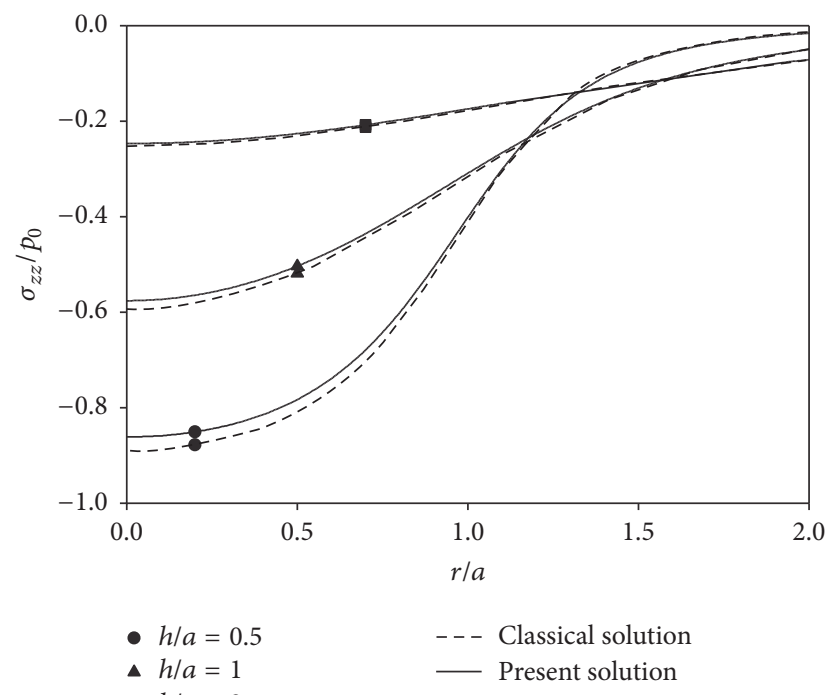

Classical solution

- $h / a=2$

(b)

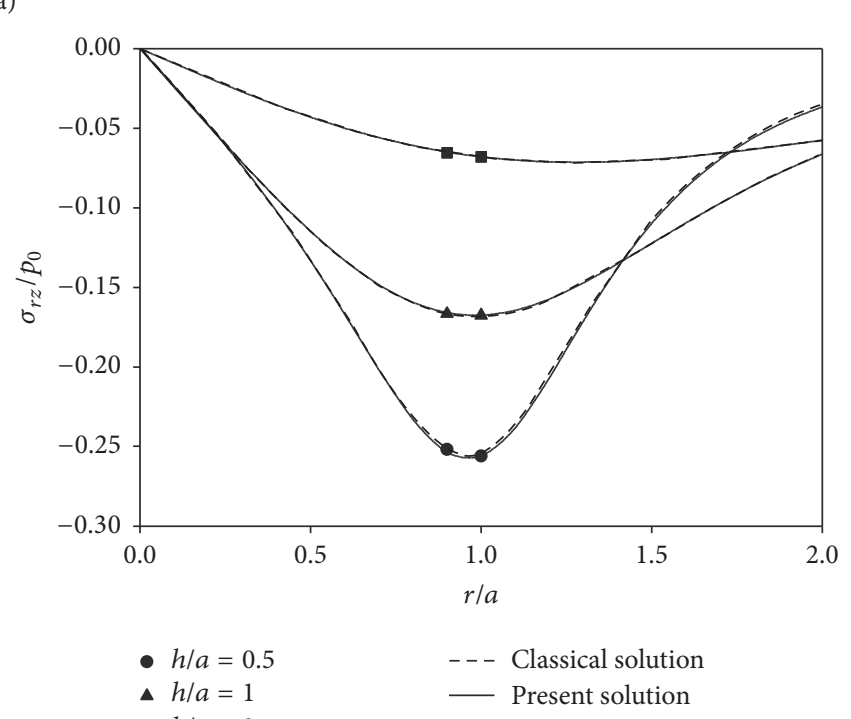

- $h / a=0.5 \quad---$ Classical solution

- $h / a=1 \quad$ Present solution

- $h / a=2 \quad \circ$ Intarit [34]

- $h / a=100$

- $h / a=1 \quad$ Present solution

- $h / a=2$

FiguRE 2: Radial variations of elastic fields under the vertical loading for different values of layer thickness $(h / a)$ : (a) surface displacements $(z=0)$ and (b) stresses at the interface $(z=h)$.

$$
\begin{aligned}
\bar{P} & =0 \\
\bar{Q}(\bar{\xi}) & =\frac{2 q_{0}}{\bar{\xi}^{2}} J_{1}(\bar{\xi} \bar{a})-\frac{q_{0} \bar{a}}{\bar{\xi}} J_{0}(\bar{\xi} \bar{a}) .
\end{aligned}
$$

In addition, the numerical results presented hereafter correspond to the case where the material for the upper layer (domain " 1 ") is Si [100] whereas Al [111] is chosen for the underlying half space (domain " 2 "), respectively. The material properties for both domains are given in Table 3 [5].

Figure 2 presents radial variations of nondimensional displacements at the top surface $(z=0)$ and nondimensional stresses at the interface $(z=h)$ of a layered elastic half space under the vertical loading for different values of normalized thickness of the top layer $(h / a)$. Note that the stress profiles
TABLE 3: Material properties employed in numerical study.

\begin{tabular}{lcc}
\hline Material parameters & Upper layer & Underlying half space \\
\hline$\lambda\left(\mathrm{N} / \mathrm{m}^{2}\right)$ & $78.0849 \times 10^{9}$ & $58.17 \times 10^{9}$ \\
$\mu\left(\mathrm{N} / \mathrm{m}^{2}\right)$ & $40.2256 \times 10^{9}$ & $26.13 \times 10^{9}$ \\
$\tau^{s}(\mathrm{~N} / \mathrm{m})$ & 0.6056 & 1 \\
$\lambda^{s}(\mathrm{~N} / \mathrm{m})$ & 4.4939 & 6.8511 \\
$\mu^{s}(\mathrm{~N} / \mathrm{m})$ & 2.7779 & -0.376 \\
$\mathcal{\kappa}^{s}(\mathrm{~N} / \mathrm{m})$ & 10.0497 & 6.0991 \\
\hline
\end{tabular}

in all figures presented in this section are computed at the interface $(z=h)$ at the bulk material of the underlying half space. Figure 2(a) shows radial profiles of vertical and 

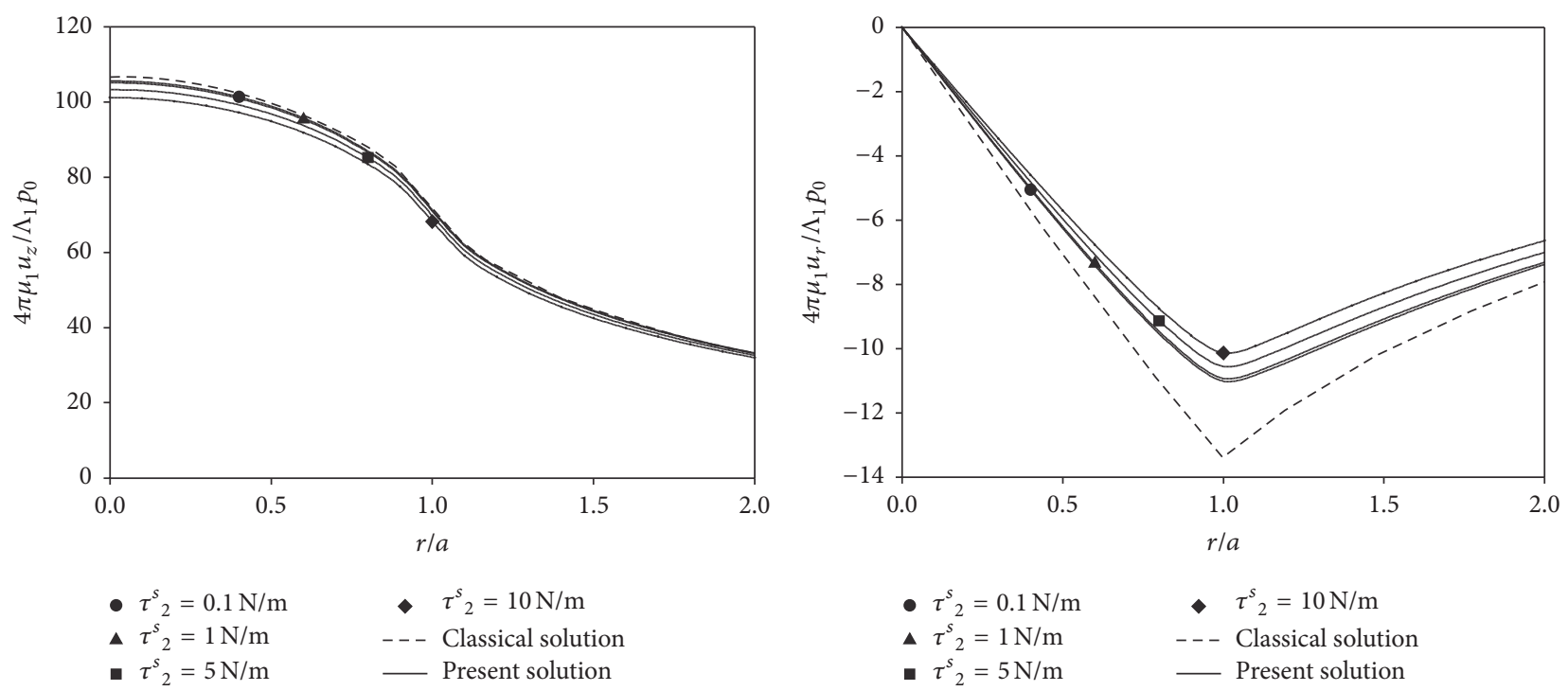

(a)
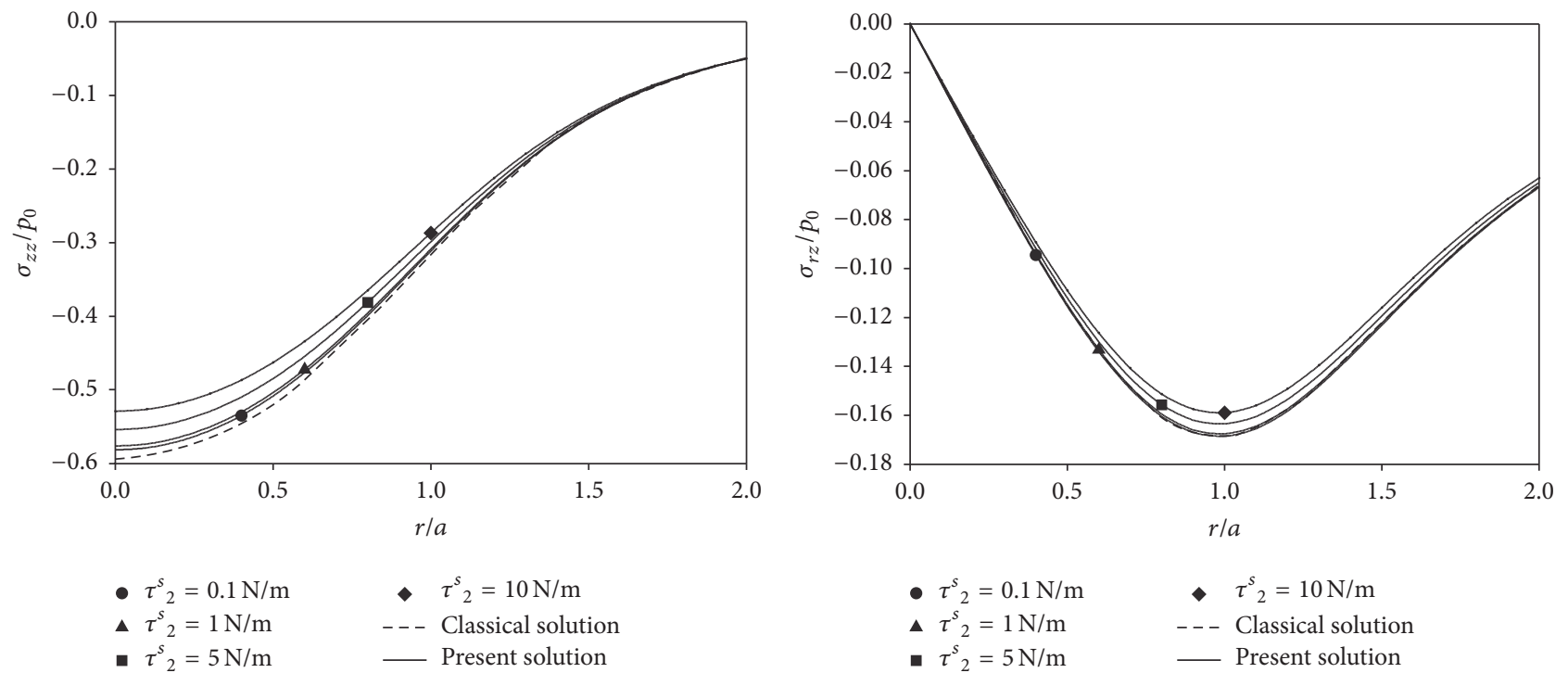

(b)

Figure 3: Radial variations of elastic fields under the vertical loading for $h / a=1$ and different magnitudes of residual surface stress $\left(\tau^{s}{ }_{2}\right):(a)$ surface displacements $(z=0)$ and (b) stresses at the interface $(z=h)$.

radial surface displacements for various values of $h / a$ whereas the profiles of normal and shear stresses at the interface are illustrated in Figure 2(b). The classical solutions, also presented in these figures for comparison, are obtained by setting the parameters associated with the surface energy effects to be zero; that is, $\tau^{\mathcal{s}} \equiv 0$ and $\kappa^{\mathcal{s}} \equiv 0$. It is evident from Figure 2 that although the results from the present study and the classical solution display similar trends for both displacements and stresses at all values of $h / a$, the surface energy effect renders the layered medium stiffer. The present solution yields lower surface displacements and stresses at the interface. The influence of surface energy is however less significant in the interface stresses, especially in the case of the shear stress. It is also found that the magnitude of all displacements and stresses decrease with increasing the normalized thickness of the layer $(h / a)$ since the upper layer is stiffer than the underlying half space (Lamé constants of Si [100] are higher than those of $\mathrm{Al} \mathrm{[111]).} \mathrm{In} \mathrm{addition,} \mathrm{as}$ the layer thickness increases both vertical and radial surface displacements move towards the homogeneous half-space solutions presented by Intarit [34], and both solutions are virtually identical when $h / a \geq 100$.

Radial profiles of normalized surface displacements $(z=$ 0 ) and normalized stresses at the interface $(z=h)$ of the layered elastic medium under the vertical loading are shown in Figure 3 to demonstrate the influence of the residual surface stress $\left(\tau^{s}\right)$ on elastic fields. The values of the residual surface stress in the underlying half space are varied 

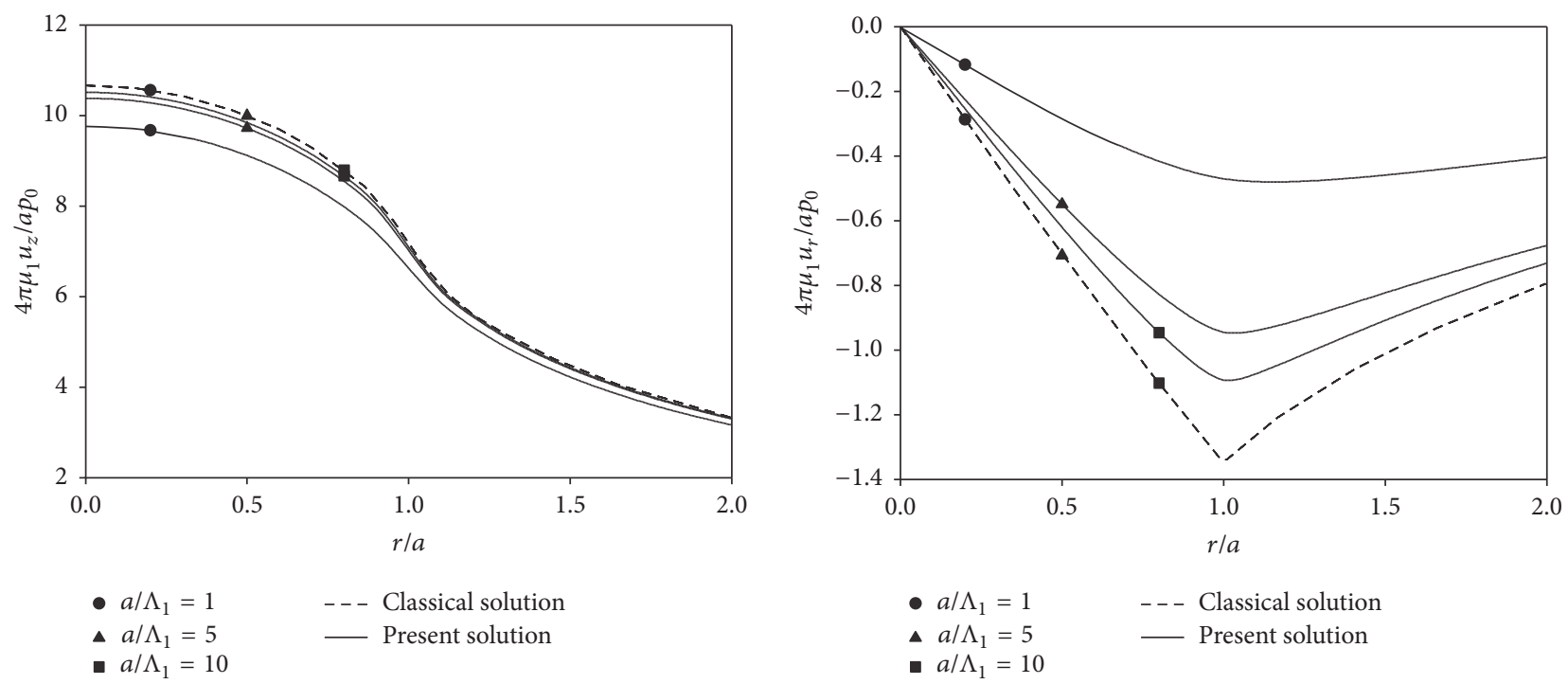

(a)
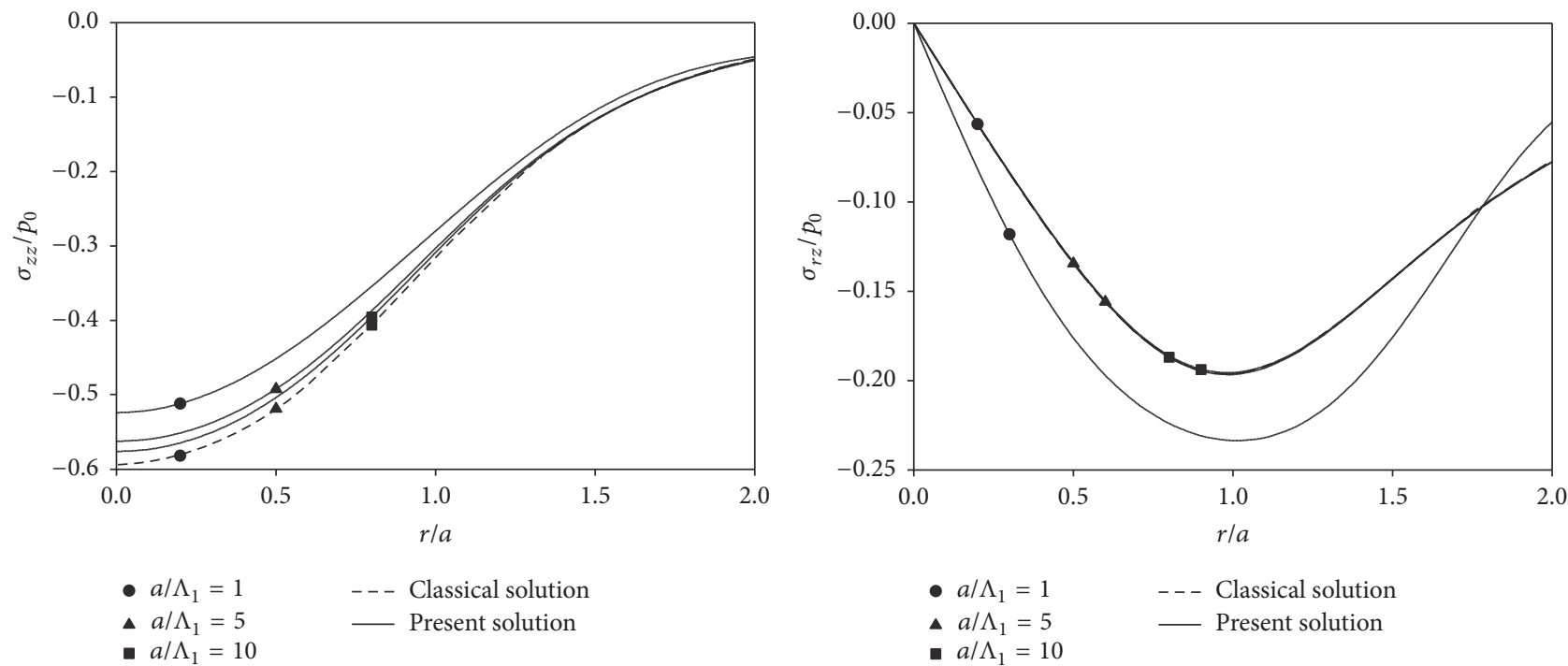

(b)

FIGURE 4: Radial variations of elastic fields under the vertical loading for $h / a=1$ and different values of loading radius ( $\bar{a})$ : (a) surface displacements $(z=0)$ and (b) stresses at the interface $(z=h)$.

(i.e., $\tau_{2}^{s}=0.1,1,5,10 \mathrm{~N} / \mathrm{m}$ ) whereas other material parameters associated with both upper layer and underlying half space given in Table 3 remain unchanged. In addition, the normalized thickness of $h / a=1$ is considered in the numerical results shown in this figure. Once again, the influence of the surface stress is clearly observed from the displacement and stress solutions presented in Figure 3. The values of all displacements and stresses from the present study are substantially reduced from their classical elasticity counterparts as the value of the residual surface stress increases.

The next numerical results are presented to demonstrate the size-dependent behavior of the present solution when the influence of surface energy effects is considered. Figure 4 shows radial variations of vertical and radial surface displacements and the vertical and shear stresses at the interface of the layered half space under the vertical loading for different values of the normalized radius of loading area $\bar{a}$ (i.e., $\left.\bar{a}=a / \Lambda_{1}=1,5,10\right)$. In addition, the thicknesses of the top layer and the circular loading area are varied while their ratio is maintained at $h / a=1$. Note that the solution when $\bar{a}=1$ corresponds to the case where the thickness of the layer is equal to the characteristic length $\left(\Lambda_{1}\right)$. The corresponding nondimensional solution for the classical elasticity case is also shown, and it is size-independent. The size-dependency of the present solution is clearly observed in all displacement and stress profiles. It is evident from the numerical results presented in Figure 4 that the present solution accounting for surface energy effects approaches the classical solution as the loading radius increases. This is consistent with the fact that a 

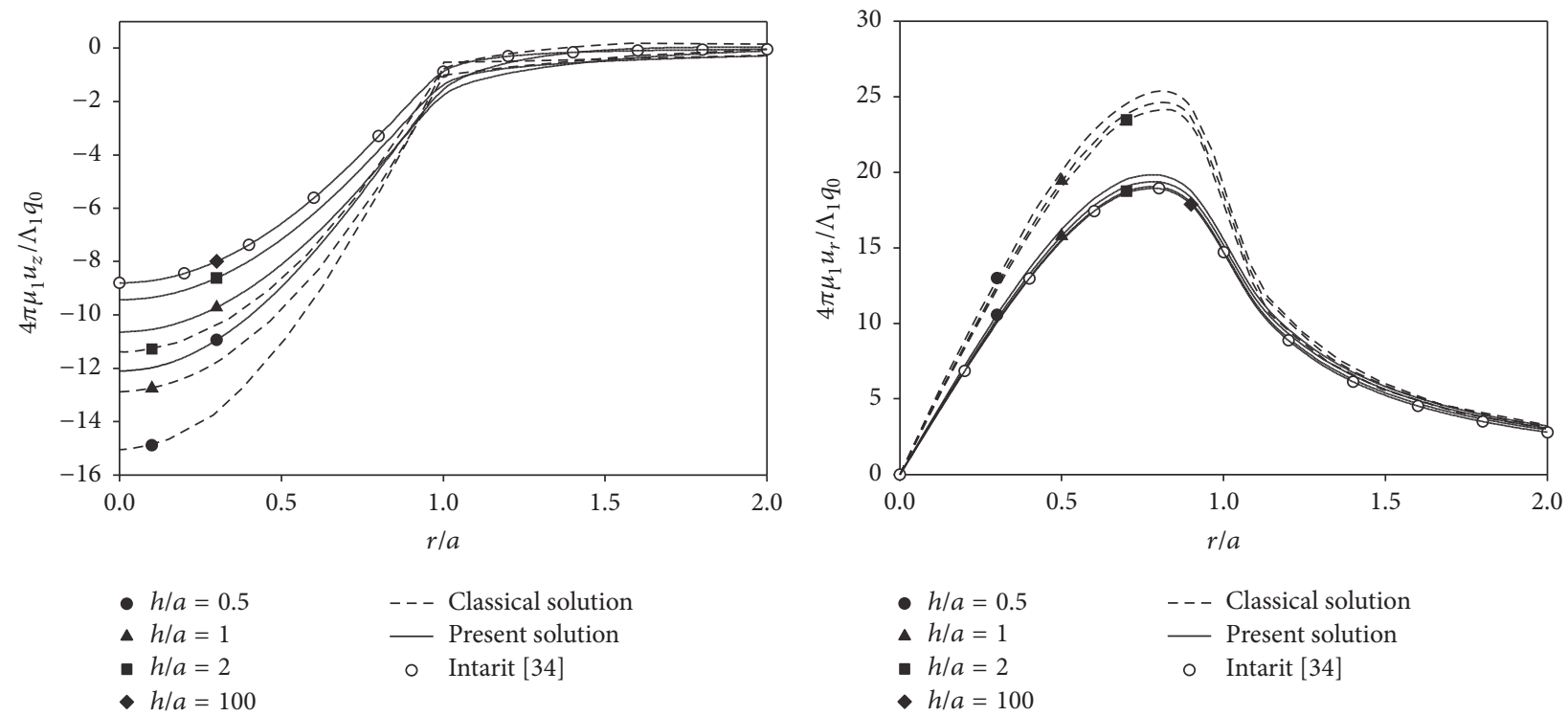

(a)
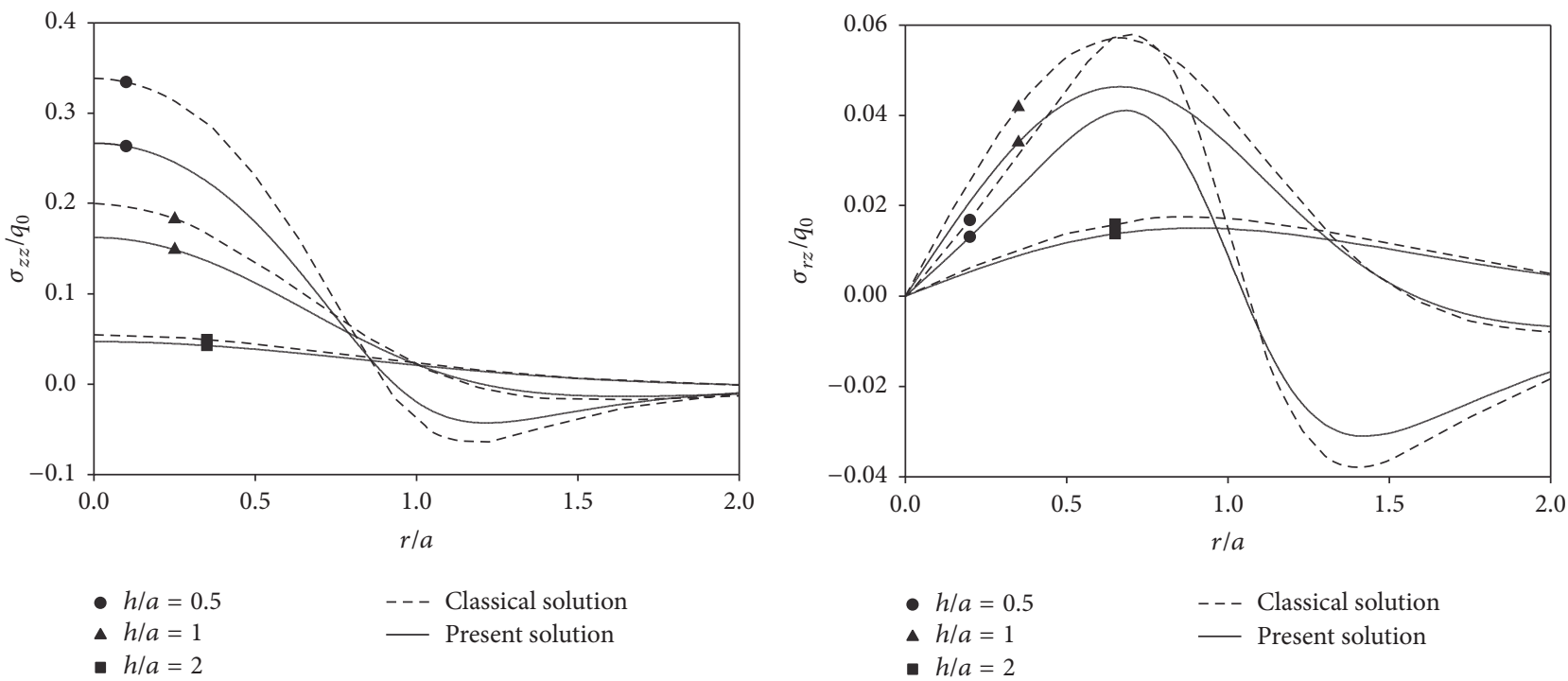

(b)

FIGURE 5: Radial variations of elastic fields under the radial loading for different values of layer thickness $(h / a)$ : (a) surface displacements $(z=0)$ and (b) stresses at the interface $(z=h)$.

larger loading area would produce higher displacements and stresses.

The final set of the numerical results correspond to the case where the layered elastic half space is subjected to the radial loading, in which the tangential traction is applied linearly distributed over a circular area of normalized radius $\bar{a}=10$. Figure 5 presents radial profiles of nondimensional displacements at the top surface $(z=0)$ and nondimensional stresses at the interface $(z=h)$ for different values of $h / a$. It is evident from Figure 5 that both displacements and stresses of the layered half space under the radial loading depend more significantly on surface energy effects for all values of $h / a$ when compared to the results presented in Figure 2 under the vertical loading case. The presence of surface stresses significantly lowers the magnitude of all displacements and stresses shown in Figure 5. In addition, all displacements and stresses are reduced as the normalized thickness of the layer $(h / a)$ increases. Once again, both vertical and radial surface displacements are practically the same as the halfspace solutions given by Intarit [34] when $h / a \geq 100$ similar to what was observed in the vertical loading case.

\section{Conclusions}

An analytical treatment of a layered elastic half space under axisymmetric surface loading, taking into account the influence of surface energy effects, is presented in this paper. The boundary value problem corresponding to a layered elastic 
half space subjected to axisymmetric normal and tangential traction is formulated based on the complete GurtinMurdoch theory of surface elasticity and the application of Hankel integral transforms. An efficient numerical quadrature scheme is developed to evaluate all involved integrals. Selected numerical results for radial profiles of displacements and stresses are presented to portray the influence of various parameters on elastic fields. The numerical results indicate that the surface energy effects play an important role in both stress and displacement fields of a layered elastic medium. Surface residual stress reduces the surface displacements and stresses at the interface of the layered medium. Unlike the classical elasticity solution, the present study shows substantial size-dependency of elastic fields. Both displacement and stress fields show strong dependency on the loading area, and the magnitudes of nondimensional displacements and stresses increase with increasing the radius of the loading area. The application of the present fundamental solution for nanoindentation and contact problems is currently underway and will be presented in a future publication. In addition, the present solution can also be used as a benchmark solution for assessing the accuracy of numerical models such as the finite element and boundary element methods, which can be used to investigate more complicated problems in the presence of surface energy effects.

\section{Competing Interests}

The authors declare that there is no conflict of interests regarding the publication of this paper.

\section{Acknowledgments}

The work presented in this paper was supported by the Thailand Research Fund (Grants no. BRG 5880017 and PHD/ 0096/2554). The support is gratefully acknowledged.

\section{References}

[1] B. Yakobson, "Nanomechanics," in Handbook of Nanoscience, Engineering and Technology, W. A. Goddard, D. W. Brenner, S. E. Lyshenski, and G. J. Iafrate, Eds., vol. 17, pp. 1-18, CRC Press, Boca Raton, Fla, USA, 2003.

[2] E. W. Wong, P. E. Sheehan, and C. M. Lieber, "Nanobeam mechanics: elasticity, strength, and toughness of nanorods and nanotubes," Science, vol. 277, no. 5334, pp. 1971-1975, 1997.

[3] M. E. Gurtin and A. I. Murdoch, "A continuum theory of elastic material surfaces," Archive for Rational Mechanics and Analysis, vol. 57, pp. 291-323, 1975.

[4] M. E. Gurtin and A. I. Murdoch, "Surface stress in solids," International Journal of Solids and Structures, vol. 14, no. 6, pp. 431-440, 1978.

[5] R. E. Miller and V. B. Shenoy, "Size-dependent elastic properties of nanosized structural elements," Nanotechnology, vol. 11, no. 3, pp. 139-147, 2000.

[6] R. Dingreville, J. Qu, and M. Cherkaoui, "Surface free energy and its effect on the elastic behavior of nano-sized particles, wires and films," Journal of the Mechanics and Physics of Solids, vol. 53, no. 8, pp. 1827-1854, 2005.
[7] R. D. Mindlin, "Micro-structure in linear elasticity," Archive for Rational Mechanics and Analysis, vol. 16, pp. 51-78, 1964.

[8] H. G. Georgiadis and D. S. Anagnostou, "Problems of the Flamant-Boussinesq and Kelvin type in dipolar gradient elasticity," Journal of Elasticity, vol. 90, no. 1, pp. 71-98, 2008.

[9] X.-L. Gao and M. Q. Liu, "Strain gradient solution for the Eshelby-type polyhedral inclusion problem," Journal of the Mechanics and Physics of Solids, vol. 60, no. 2, pp. 261-276, 2012.

[10] X.-L. Gao and S.-S. Zhou, "Strain gradient solutions of halfspace and half-plane contact problems," Zeitschrift für angewandte Mathematik und Physik, vol. 64, no. 4, pp. 1363-1386, 2013.

[11] G.-Y. Huang and S.-W. Yu, "Effect of surface elasticity on the interaction between steps," Journal of Applied Mechanics, vol. 74, no. 4, pp. 821-823, 2007.

[12] X. J. Zhao and R. K. Rajapakse, "Analytical solutions for a surface-loaded isotropic elastic layer with surface energy effects," International Journal of Engineering Science, vol. 47, no. 11-12, pp. 1433-1444, 2009.

[13] P. Intarit, T. Senjuntichai, and R. K. N. D. Rajapakse, "Dislocations and internal loading in a semi-infinite elastic medium with surface stresses," Engineering Fracture Mechanics, vol. 77, no. 18, pp. 3592-3603, 2010.

[14] D. X. Lei, L. Y. Wang, and Z. Y. Ou, "Elastic analysis for nanocontact problem with surface stress effects under shear load," Journal of Nanomaterials, vol. 2012, Article ID 505034, 7 pages, 2012.

[15] A. A. Abdel Rahman and F. F. Mahmoud, "Analysis of nanocontact problems of layered viscoelastic solids with surface energy effects under different loading patterns," Acta Mechanica, vol. 227, no. 2, pp. 527-548, 2016.

[16] Z.-Q. Wang, Y.-P. Zhao, and Z.-P. Huang, "The effects of surface tension on the elastic properties of nano structures," International Journal of Engineering Science, vol. 48, no. 2, pp. 140-150, 2010.

[17] P. Intarit, T. Senjuntichai, J. Rungamornrat, and R. Rajapakse, "Surface elasticity and residual stress effect on the elastic field of a nanoscale elastic layer," Interaction and multiscale mechanics, vol. 4, no. 2, pp. 85-105, 2011.

[18] J. Rungamornrat, P. Tuttipongsawat, and T. Senjuntichai, "Elastic layer under axisymmetric surface loads and influence of surface stresses," Applied Mathematical Modelling. Simulation and Computation for Engineering and Environmental Systems, vol. 40, no. 2, pp. 1532-1553, 2016.

[19] S. Zhou and X.-L. Gao, "Solutions of half-space and half-plane contact problems based on surface elasticity," Zeitschrift für angewandte Mathematik und Physik, vol. 64, no. 1, pp. 145-166, 2013.

[20] Y. Pinyochotiwong, J. Rungamornrat, and T. Senjuntichai, "Rigid frictionless indentation on elastic half space with influence of surface stresses," International Journal of Engineering Science, vol. 71, pp. 15-35, 2013.

[21] M. A. Attia and F. F. Mahmoud, "Analysis of nanoindentation of functionally graded layered bodies with surface elasticity," International Journal of Mechanical Sciences, vol. 94-95, pp. 3648, 2015.

[22] S. Azizi, B. Safaei, A. M. Fattahi, and M. Tekere, "Nonlinear vibrational analysis of nanobeams embedded in an elastic medium including surface stress effects," Advances in Materials Science and Engineering, vol. 2015, Article ID 318539, 7 pages, 2015. 
[23] Y. Sapsathiarn and R. K. N. D. Rajapakse, "Finite-element modeling of circular nanoplates," Journal of Nanomechanics and Micromechanics, vol. 3, no. 3, pp. 59-66, 2013.

[24] T. B. Nguyen, J. Rungamornrat, and T. Senjuntichai, "Analysis of planar cracks in 3D elastic media with consideration of surface elasticity," International Journal of Fracture, vol. 202, no. 1, pp. 51-77, 2016.

[25] P. Intarit, T. Senjuntichai, J. Rungamornrat, and R. K. Rajapakse, "Penny-shaped crack in elastic medium with surface energy effects," Acta Mechanica, vol. 228, no. 2, pp. 617-630, 2017.

[26] L. H. He and C. W. Lim, "Surface Green function for a soft elastic half-space: influence of surface stress," International Journal of Solids and Structures, vol. 43, no. 1, pp. 132-143, 2006.

[27] C. M. Gerrard, "Tables of stresses, strains and displacements in two-layer elastic systems under various traffic loads," Australian Road Research Board-Special Report 3, 1969.

[28] D. M. Burmister, "The general theory of stresses and displacements in layered systems. I," Journal of Applied Physics, vol. 16, no. 2, pp. 89-94, 1945.

[29] P. K. Gupta and J. A. Walowit, "Contact stresses between an elastic cylinder and a layered elastic solid," Journal of Lubrication Technology, vol. 96, no. 2, pp. 250-257, 1974.

[30] A. Perriot and E. Barthel, "Elastic contact to a coated halfspace: effective elastic modulus and real penetration," Journal of Materials Research, vol. 19, no. 2, pp. 600-608, 2004.

[31] Y. Z. Povstenko, "Theoretical investigation of phenomena caused by heterogeneous surface tension in solids," Journal of the Mechanics and Physics of Solids, vol. 41, no. 9, pp. 1499-1514, 1993.

[32] I. N. Sneddon, Fourier Transforms, McGraw-Hill, New York, NY, USA, 1951.

[33] R. Piessens, Quadpack: A Subroutine Package for Automatic Integration, Springer, Berlin, Germany, 1983.

[34] P. Intarit, Solutions of elastic medium with surface stress effects [Ph.D. thesis], Chulalongkorn University, Bangkok, Thailand, 2012. 

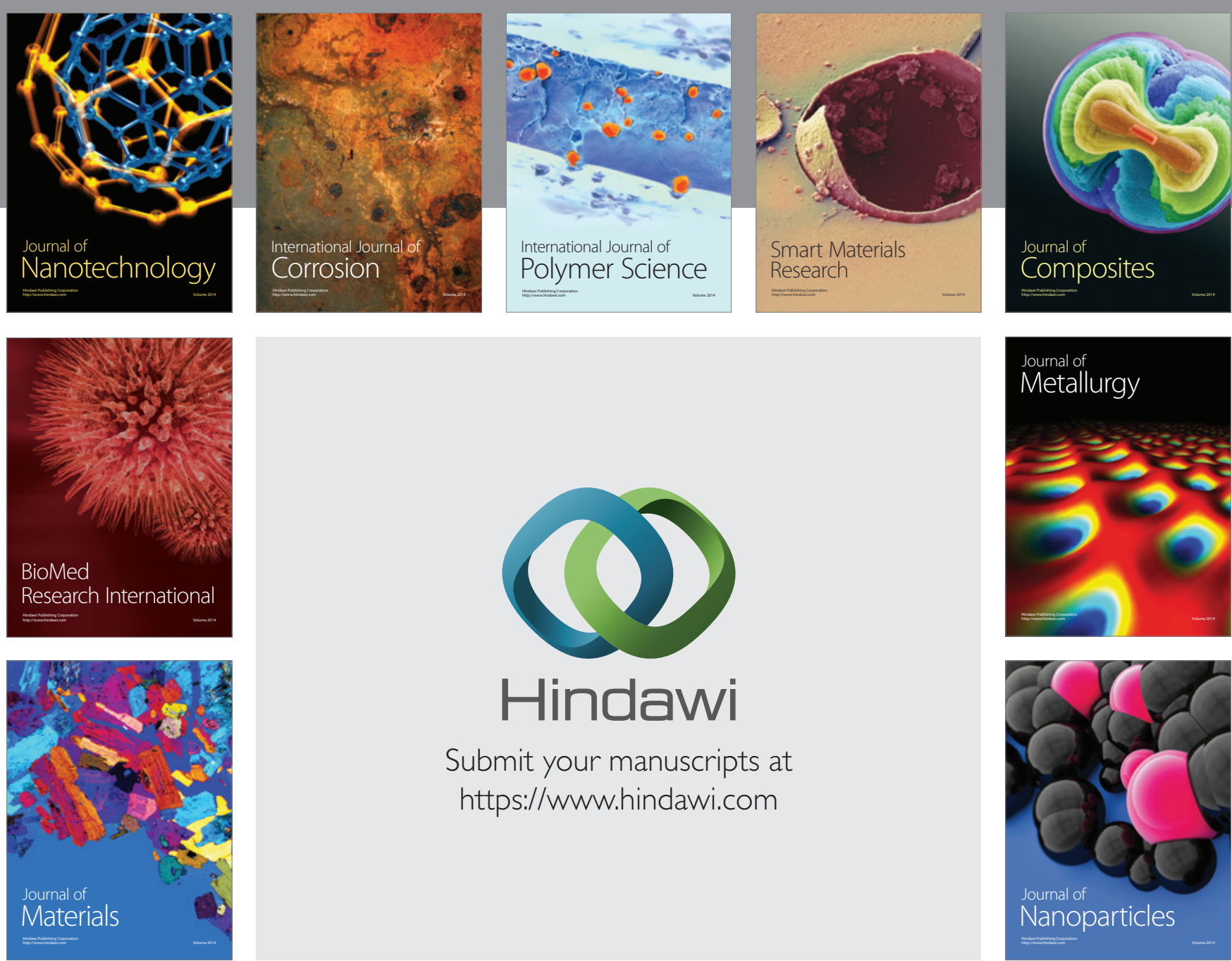

\section{Hindawi}

Submit your manuscripts at

https://www.hindawi.com

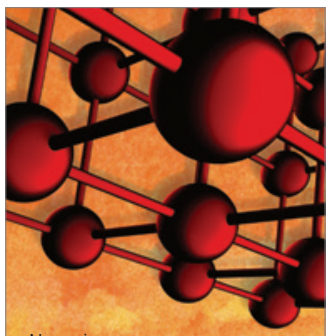

Materials Science and Engineering
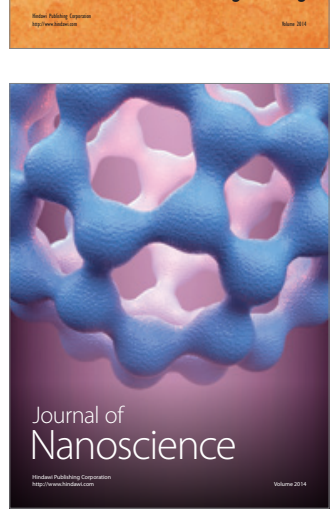
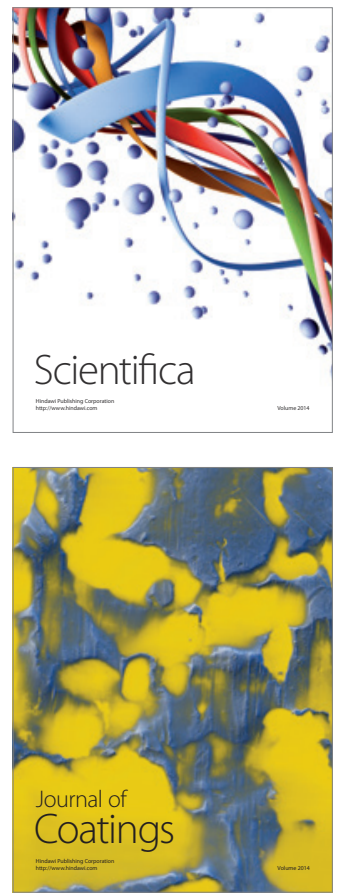
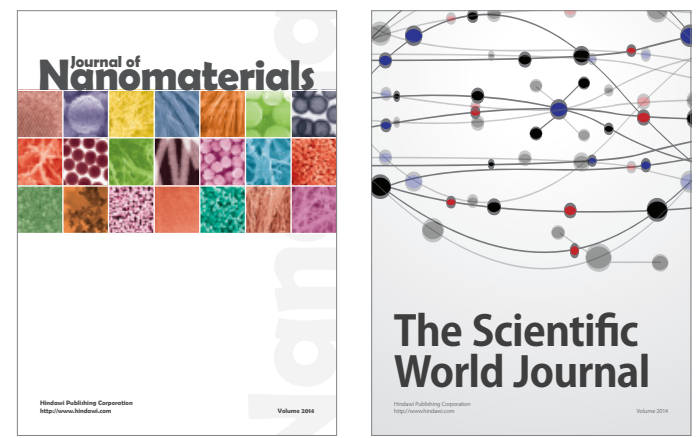

The Scientific World Journal
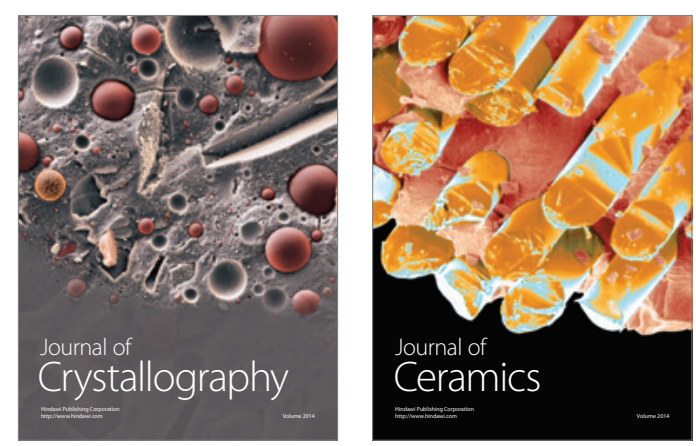
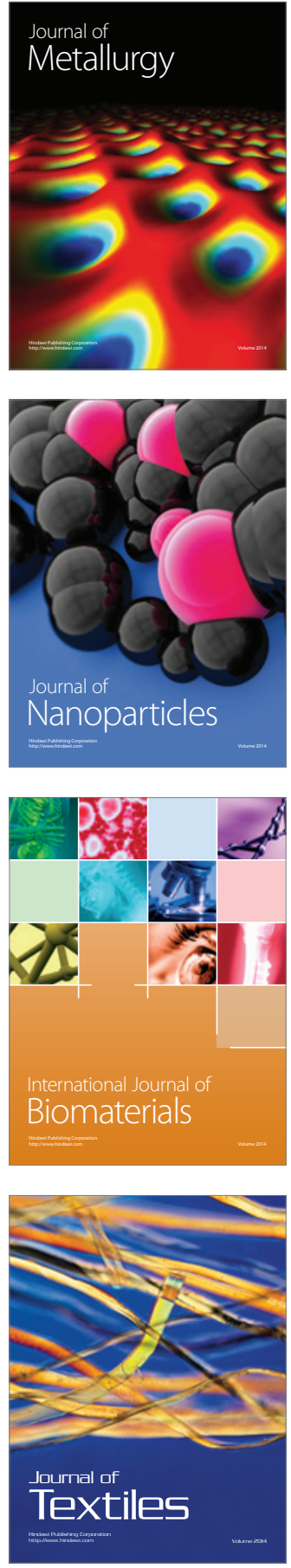